\title{
Dragon-I injector based on the induction voltage adder technique
}

\begin{abstract}
Zhang Kaizhi, Wen Long, Li Hong, Dai Zhiyong, Wang Wendou, Zhang Wenwei, Wang Meng, Li Jin, Yang Anming, Xie Yutong, Chen Sifu, Wang Huacen, Dai Guangsen, Shi Jinshui, Zhang Linwen, Deng Jianjun, and Ding Bonan

Institute of Fluid Physics, CAEP, Mianyang, Sichuan, 621900, China

(Received 7 October 2005; revised manuscript received 18 December 2005; published 15 August 2006)

The Dragon-I injector based on the induction voltage adder technique is introduced. Twelve ferrite loaded induction cells are connected in a series through central conducting stalks to achieve a pulsed voltage higher than 3.5 MV across the diode. Electrons are extracted from the velvet emitter and guided through the anode pipe by the magnets placed inside the cathode and anode shrouds. Measurements at the exit of injector show that, with an electric field of $200 \mathrm{kV} / \mathrm{cm}$ near the velvet surface and suitable magnetic field distribution, an electron beam up to $2.8 \mathrm{kA}$ can be obtained with a normalized emittance of $1040 \pi \mathrm{mm}$ mrad, and energy spread of $2.1 \%(3 \sigma)$ around the central energy of $3.5 \mathrm{MeV}$.
\end{abstract}

DOI: 10.1103/PhysRevSTAB.9.080401

\section{INTRODUCTION}

The induction voltage adder (IVA) technique is a method of connecting induction cells in series by a central metal cylinder so that their voltages are added. The summed voltages are enclosed with a slightly larger radius, generally the bore of cells. The IVA technique is widely used in high current accelerators and pulsed-power facilities [1].

Most linear induction accelerator (LIA) injectors are based on the IVA technique, such as those of Flash X-ray Radiography (FXR) [2] and Advanced Test Accelerator (ATA) [3] at LLNL, and Linear Induction Accelerator Xray Facility (LIAXF) [4] at CAEP, China. Normally, the energy of the output electron beam of this type of injector is below $2.5 \mathrm{MeV}$, mainly limited by the cantilever supporting the cathode and the anode, and by the high voltage insulation design as well, since serious sag of the longer central conducting stalk resulting from higher energy will degrade the property of the injector, and practical design is hampered by the larger radius needed to enclose the higher summed voltage at the same time.

In recent years diodes directly powered by the pulsed forming line (PFL) have come into operation at relatively higher voltage, like injectors of DARHT-I [5] at LANL and AIRIX [6] in France, and their cathodes are supported by radial insulators.

In the Dragon-I injector, radial insulators are installed in place of the cantilever, and some newly developed techniques are introduced. As we will show, the Dragon-I injector's operating energy is increased to $3.5 \mathrm{MeV}$ while the beam properties are improved at the same time.

\section{OVERVIEW OF THE INJECTOR}

The Dragon-I accelerator is a $20 \mathrm{MeV}, 2.5 \mathrm{kA}, 90 \mathrm{~ns}$ (FWHM) LIA operating in single pulse mode [7]. The output electron beam from the accelerator is expected to be focused to a spot size less than $1.5 \mathrm{~mm}$ (FWHM), which requires the output beams from the injector to have a
PACS numbers: 52.59.Mv, 29.17.+w, 29.25.Bx, 52.59.Sa

normalized emittance of $1000 \pi \mathrm{mm}$ mrad and an energy spread of $2 \%$.

Figure 1 is a block diagram of the Dragon-I injector configuration that utilizes the IVA architecture. Seven upstream induction cells and 5 downstream induction cells to the diode are connected in series, respectively, by conducting stalks. A 3.5 MV pulse that lasts for approximately $90 \mathrm{~ns}$ (FWHM) can be generated across the cathode-anode gap. When the gap is set at $170 \mathrm{~mm}$, an electron beam of $3.4 \mathrm{kA}$ can be extracted from the velvet emitter of $70 \mathrm{~mm}$ in diameter.

The injector is set on an adjustable platform with 3 degrees of freedom for precision alignment [8]. Using the pulse-wire method [9], the offset of the magnetic axis from the mechanic axis of the injector was minimized to less than $0.2 \mathrm{~mm}$, and the tilt less than $5 \mathrm{mrad}$.

The power supply units for the injector section take nearly the same design as those used to power the accelerating section of the Dragon-I accelerator [7]. A typical pulsed-power module is shown in Fig. 2. The injector uses 2 Marx generators and 12 Blumlein PFLs. The Blumlein PFLs are $45 \mathrm{~ns}$ in electric length and can be charged to $250 \mathrm{kV}$ in about $3.5 \mu \mathrm{s}$ by the Marx generator. Each induction cell is fed by one Blumlein PFL through two

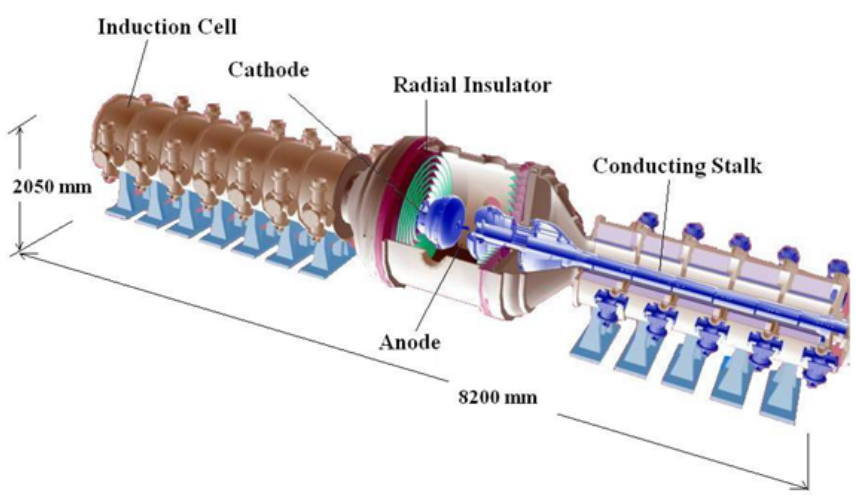

FIG. 1. (Color) Scheme of the injector of Dragon-I accelerator. 


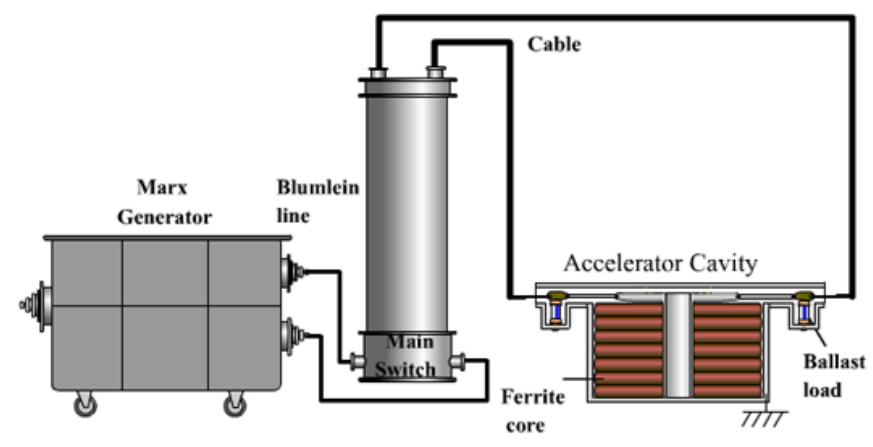

FIG. 2. (Color) Single module prototype of the pulsed-power system.

$24 \Omega$, $400 \mathrm{kV}$ coaxial cables, which are impedance matched to the $12 \Omega$ Blumlein PFL. The inductive pulse voltage can be obtained across each induction gap according to Eq. (1),

$$
V_{C}=\frac{2 V_{B}\left(Z_{F}\left\|Z_{C}\right\| Z_{L}\right)}{Z_{B}+Z_{F}\left\|Z_{C}\right\| Z_{L}}
$$

where $V_{C}(300 \mathrm{kV})$ represents the inductive voltage, $V_{B}$ and $Z_{B}$ are the output voltage and impedance from Blumlein, respectively, $Z_{C}(80 \Omega)$ is the ballast resistor, $Z_{F} \approx 180 \Omega$ is the resistance of the cell filled with ferrite, and $Z_{L} \approx 100 \Omega$ is the load impedance.

Figure 3 is the electrical schematic of the injector for the cathode side.

\section{DIODE DESIGN}

\section{A. Operating voltage}

Because of the space charge effect, the emittance of the low-energy, high intensity electron beam increases remarkably inside the injector. To decrease the space change effect, the operating voltage across the diode is increased to effectively shorten the drift time at low energy.

The dependence of emittance on energy was derived by Caporaso [10], and it might be simplified as follows:

$$
\varepsilon_{n f}^{2}=\varepsilon_{n i}^{2}+\eta_{P} / \beta \gamma,
$$

where $\varepsilon_{n i}$ is the initial normalized root mean square (rms) electron beam emittance, and $\varepsilon_{n f}$ is the rms emittance at the injector exit and defined as

$$
\varepsilon_{n}=\beta \gamma R\left(v_{\perp}^{2}-R^{\prime 2}-L^{2} / R^{2}\right) .
$$

In Eq. (3), $R$ is the rms radius, $R^{\prime}$ is the velocity of the rms radius, $v_{\perp}$ is the rms transverse velocity, and $L$ is the mechanical angular momentum of the beam. $\beta=v_{\|} / c$, where $v_{\|}$is the longitudinal velocity, and $c=3 \times$ $10^{8} \mathrm{~m} / \mathrm{s}, \gamma=\left(1-\beta^{2}\right)^{-1 / 2}$ refers to the energy as usual. $\eta_{P}$ is mainly related to the beam profile and determined by space charge effect. It is positive because a beam always evolves from an initial flat profile to a Gaussian distribution, so the increase of the emittance will be inversely proportional to " $\beta \gamma$ " [the second term on the right-hand side of Eq. (2)], as shown in Eq. (4):

$$
\Delta \varepsilon_{n}^{2}=\eta_{P} / \sqrt{\gamma^{2}-1}
$$

As shown in Fig. 4, that in the lower energy region the emittance growth will decrease rapidly with energy as voltage increases, however there is no need to get higher energy, since $\Delta \varepsilon_{n}{ }^{2}$ reduces to less than $0.02 * \eta_{P}$ when $\gamma$ increases from 7 to 8 . Furthermore, the beam is dominated by emittance after $\gamma$ rises to a relatively high value. That is the reason why we choose $3.5 \mathrm{MV}$ as the operating voltage applied to the diode.

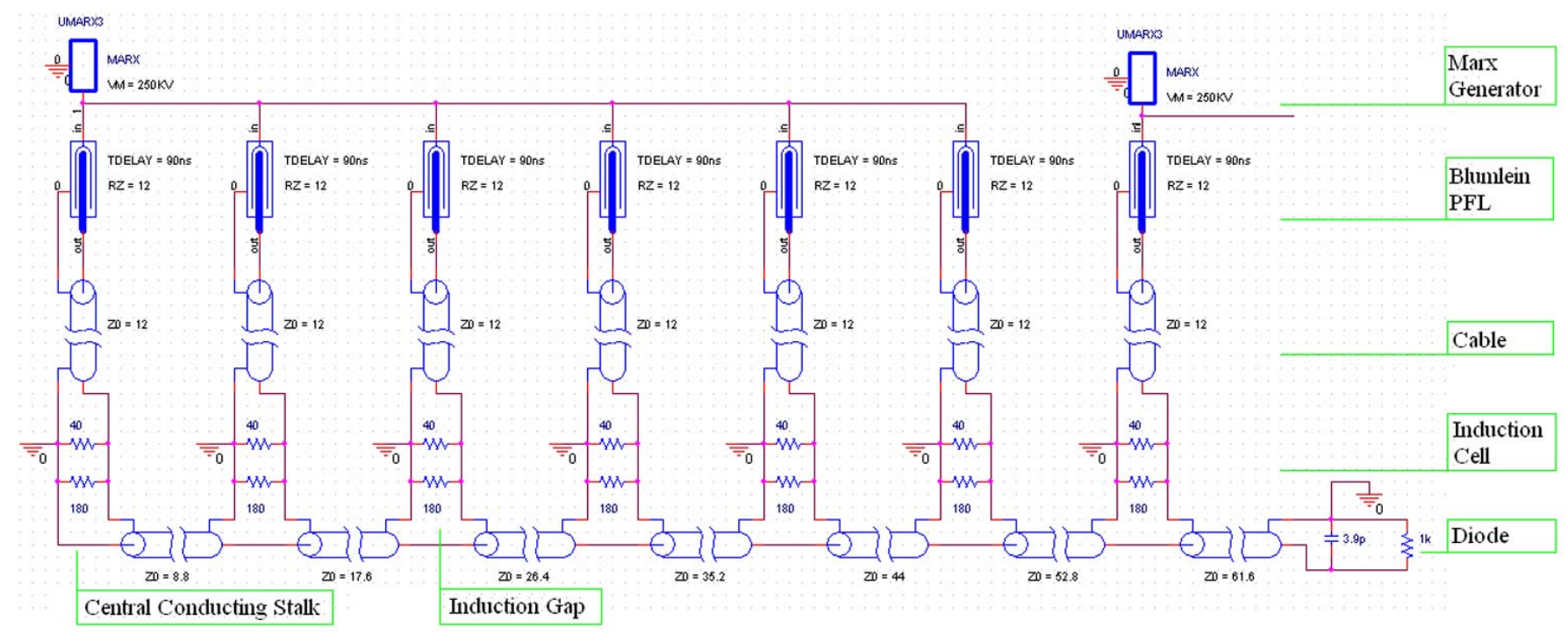

FIG. 3. (Color) Electrical schematic diagram of the injector (cathode side). 


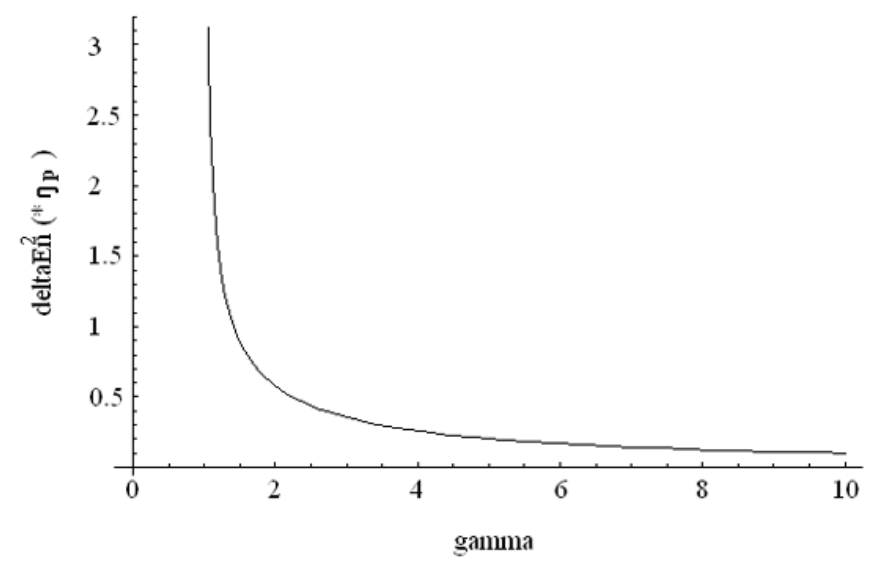

FIG. 4. Dependency of emittance evolution on energy.

\section{B. Diode configuration}

The configuration of the diode and the simulated electric field map are shown in Fig. 5.

Large flat diode electrodes of $150 \mathrm{~mm}$ in radius were chosen to optimize the electric field distribution in the diode zone. The radius of the anode aperture was optimized to be $60 \mathrm{~mm}$ according to numerical simulation, while $100 \mathrm{~mm}$ downstream from the anode electrode the radius of the anode pipe increases to $80 \mathrm{~mm}$ to accommodate the
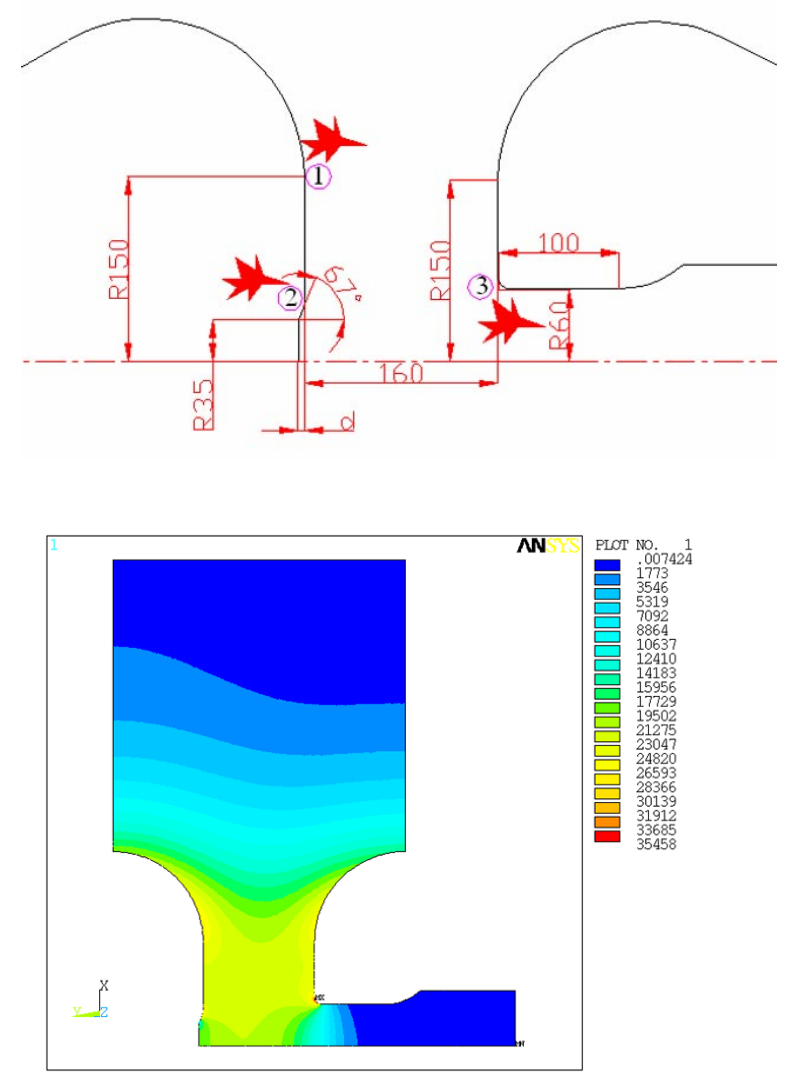

FIG. 5. (Color) Drawing of the configuration (top) and electric field map (bottom) of the diode.

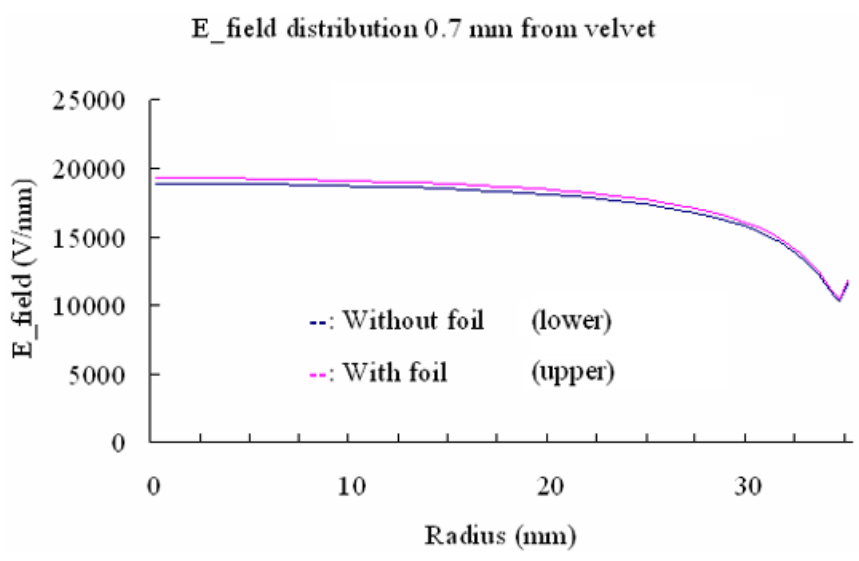

FIG. 6. (Color) The effect of anode foil on the field distribution near emitter surface.

increasing beam envelope. The cathode-to-anode (AK) gap is adjustable from 150 to $175 \mathrm{~mm}$, and this large AK gap helps to weaken the effect of the anode aperture on the field distribution near the emitter surface, so that a foilless anode is acceptable. The difference of the simulated field distribution between diodes with and without foil is shown in Fig. 6, and it verified the above conclusion.

Since the uniformity of the field distribution across the diode is of high importance, a foil was placed in the front of the anode aperture in former injectors, such as those of FXR and LIAXF. But the lifetime of a normal foil is less than 100 shots, and in addition the foil scatters and focuses the particles passing through, leading to beam quality degradation.

Velvet cloth was chosen as the emitting material due to its uniform emission and relatively low electric field threshold for high intensity emission with high brightness [11].

A recessed cathode structure was adopted with the cathode surface of $67.5^{\circ}$ around the emitting material, like Pierce cathode [12], as shown in Fig. 5. The radial electric field created by this recessed structure will balance the outward expansion of emitted electron beam due to the space charge effect. The recess depth of the flat cathode surface, " $d$ " in Fig. 5, is an important parameter which determines the effectiveness of such structure. The best value is $6 \mathrm{~mm}$, which was demonstrated in the simulation of beam dynamics with the angle maintained as the depth varying, as shown in Table I, where the emittance is acquired at the exit of the injector.

TABLE I. Normalized emittance relative to different recess depths of the emitter $\left(V_{\mathrm{AK}}=3.6 \mathrm{MV} ; D_{\mathrm{AK}}=170 \mathrm{~mm}\right)$.

\begin{tabular}{lccccc}
\hline \hline Recess depth $(\mathrm{mm})$ & 0 & 2 & 4 & 6 & 8 \\
\hline $\begin{array}{l}\text { Normalized } \\
(\text { Simulated, } \pi \text { mm mrad })\end{array}$ & & & & & \\
\hline \hline
\end{tabular}


There are 3 peak points labeled (1), (2), and (3) in the diode zone in Fig. 5, and the corresponding electric fields are 273,281 , and $350 \mathrm{kV} / \mathrm{cm}$ when the cathode-anode gap is $170 \mathrm{~mm}$, and the diode voltage is 3.6 MV. Because of fine surface buffing, unwanted electron emission from the metallic surface of the cathode is negligible.

\section{INDUCTION CELL}

Induction cells of the injector were modified from those of the accelerating section [13], with new features added to achieve better high voltage performance.

The electric pressure inside the induction cell adjacent to the cathode is the highest where high voltage up to $2.1 \mathrm{MV}$ is applied, and its configuration reveals some basic approaches in the cell design.

The inner diameter of bore of the induction cell, $D_{b}$, and the outer diameter of the central conducting stalk, $D_{s}$, should follow the optimum principle, $D_{b} / D_{s}=\mathrm{e} \cong$ 2.7183. When $D_{b}$ is $490 \mathrm{~mm}, D_{s}$ should be $180 \mathrm{~mm}$ to damp the peak field below an experiential critical value, $225 \mathrm{kV} / \mathrm{cm}$.

On account of the impedance matching between neighboring section of coaxial lines which are mainly composed of bore of the cell and the stepped stalk, $D_{s}$ was modified from the optimum value and fixed at $129 \mathrm{~mm}$.

The width of the induction gap as shown in Fig. 7 was optimized to obtain desired field distribution, and $38 \mathrm{~mm}$ was taken finally. Furthermore, the inner diameter of the cover board of the induction gap was enlarged to $530 \mathrm{~mm}$ to depress the peak electric field along the conducting stalk.

In order to achieve high voltage performance, in addition to surface polishing, the cells are filled with insulating oil with relative permittivity of 2.3. Simulation of the final design shows that the peak field along the conducting stalk is $213.4 \mathrm{kV} / \mathrm{cm}$, and that around the induction gap is $171.3 \mathrm{kV} / \mathrm{cm}$.

All the cells took nearly the same design except for the cover board, and their dimensions are shown in Fig. 7.

Because transportation magnets were added inside the ferrite torroids, the bore diameter of cells downstream to the anode was reduced to $412 \mathrm{~mm}$. The simulated peak field strength is $198.5 \mathrm{kV} / \mathrm{cm}$ along the anode stalk, and $180.7 \mathrm{kV} / \mathrm{cm}$ near the induction gap, when the applied voltage is $1.5 \mathrm{MV}$ between the bore and the stalk.

Thirteen ferrite torroids are placed inside each cell to generate an inductive voltage pulse of $300 \mathrm{kV}, 90 \mathrm{~ns}$. The ferrite torroid is probably the largest one-piece torroid in dimension in the world, and was manufactured in Jiuyuan Plant of magnetic material, Science city of Sichuan Province [14], with $510 \mathrm{~mm}$ inner diameter, $800 \mathrm{~mm}$ outer diameter, thickness of $23 \mathrm{~mm}$, and flux swing larger than $0.72 \mathrm{~T}$.

\section{MAGNETIC GUIDING SYSTEM IN THE DIODE ZONE}

The beam transportation system includes 5 magnets inside the anode side induction cells and 3 magnets in the diode zone, including the bucking magnet, the extracting magnet, and the bridge magnet. Each magnet consists of a solenoid and a pair of printed board dipole trim magnets [15] except the bucking magnet where the trim magnet is absent. The extracting solenoid is divided into two parts to make it convenient to tune the magnetic field distribution, as shown in Fig. 8.

Traditionally, the diode magnets are placed outside the vacuum chamber [2-4]. The diode of the Dragon-I injector is inside a chamber with the inner radius up to $1400 \mathrm{~mm}$ to
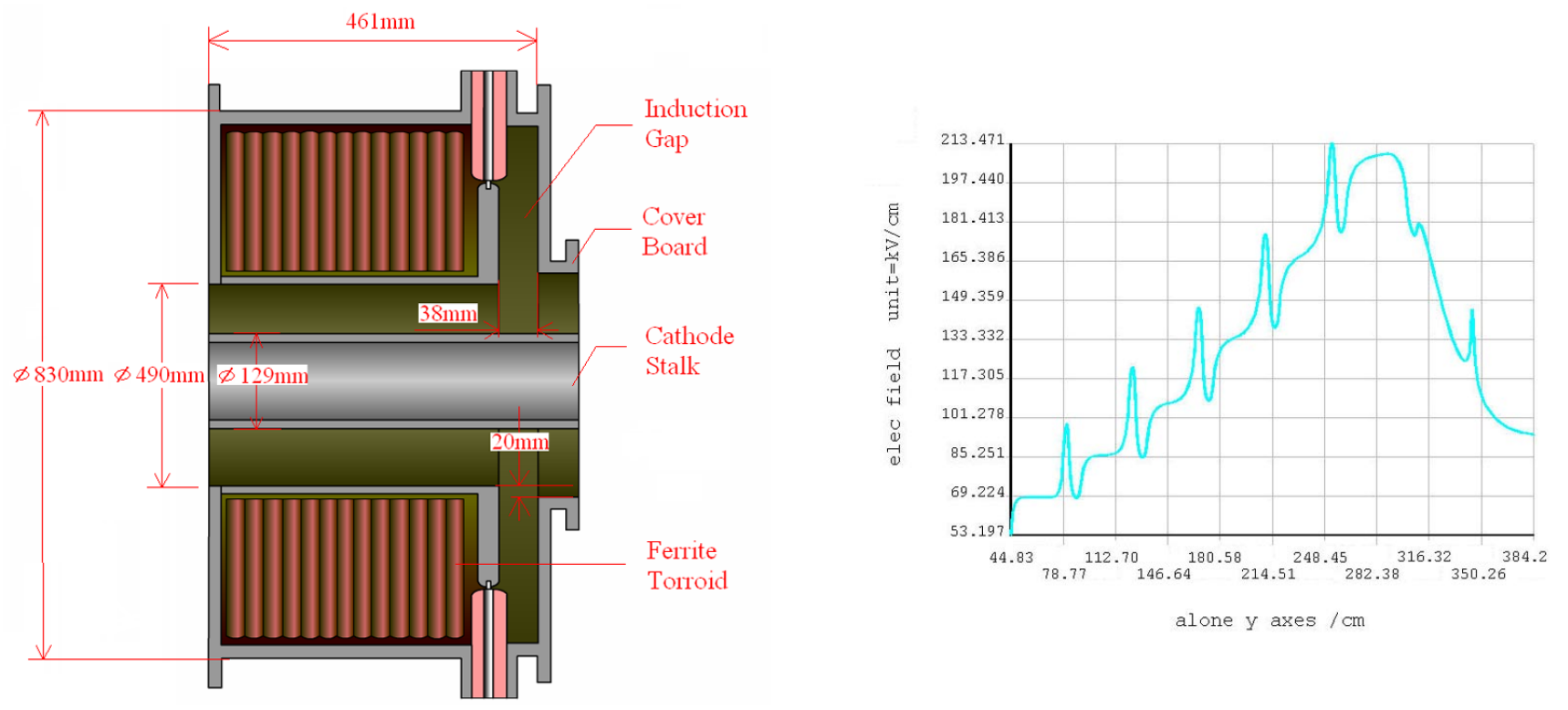

FIG. 7. (Color) Cut-away view of the cell adjacent to cathode (left) and field distribution along cathode stalk (right). 


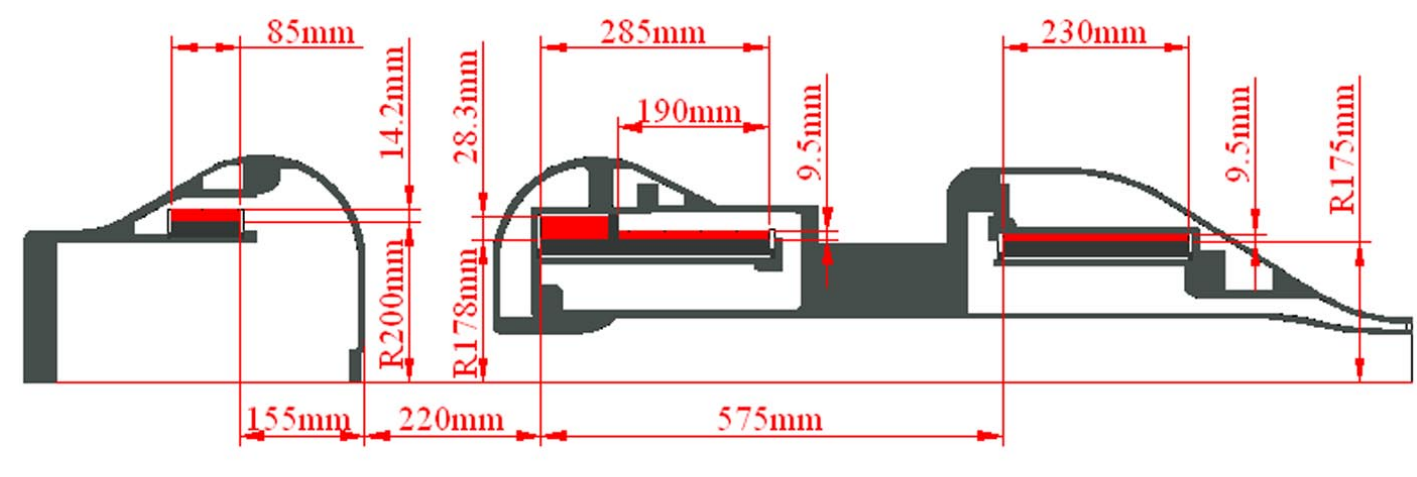

Bucking Magnet Extracting Magnet Bridge Magnet

FIG. 8. (Color) Scheme of the magnets in the diode zone.

protect the radial insulators from possible high voltage damage, and this had been a great challenge in designing magnetic guiding system in the diode zone. It is difficult not only to manufacture and to align such large and thin magnets, but to energize them as well.

In order to solve the above-mentioned puzzles, these magnets are placed inside the cathode and anode shroud, respectively, as shown in Fig. 8. The 3 solenoids are bifilar coils [16] wound with insulated, $2.54 \mathrm{~mm}$ diameter, copper wire. The trim magnets are attached to the inner bore of the solenoids.

These magnets are placed inside an air chamber sealed from the surrounding vacuum environment. Air chambers with acute angles and flanges are contained inside field shaping shells in order to be protected from high voltage damage.

The path of the magnet leads is of high importance since the leading wires have to go through the cathode and anode pipe, and any visible effect on beam transportation is unacceptable.

A dual-layer anode pipe was put forward as shown in Fig. 9, and the air-filled interlayer can accommodate the power feed wires of the extracting and bridge magnets. Twenty-four wires are divided into 12 couples with opposite currents, and distributed axisymmetrically around the circumference $30^{\circ}$ apart. Simulation shows that the magnetic field generated by leading wires in operation condition is less than $5 \times 10^{-5} \mathrm{~T}$, only $0.1 \%$ of the applied

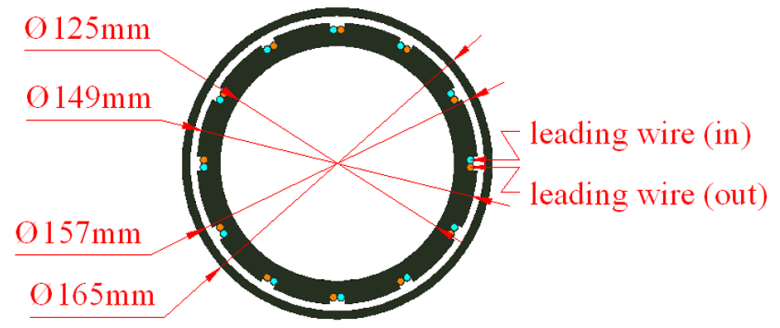

FIG. 9. (Color) Cut-away view of the dual-layer anode pipe. transportation field, so that the effect of leading wires on the beam transportation is negligible.

The leading wires of the dipole trim magnets go through the hollow space in a similar way.

The leading wires of the bucking magnets go through the hollow cathode stalk directly.

\section{CENTRAL CONDUCTING STALK}

The central conducting stalks behave like the inner conductor of coaxial transmission lines, with the bore of the induction cell as the outer one.

Voltage pulses input from every gap of the cells are summed up and transmit along the stalks, and the pulses will be reflected or pass through at every gap based on the following rules:

$$
\begin{aligned}
U_{\text {tra }} & =\frac{2 R_{d}}{R_{d}+R_{\text {cell }}+R_{u}} U_{\mathrm{in}}, \\
U_{\text {ref }} & =\frac{R_{d}+R_{\text {cell }}-R_{u}}{R_{d}+R_{\text {cell }}+R_{u}} U_{\mathrm{in}},
\end{aligned}
$$

where $U_{\text {in }}$ represents the incident wave, $U_{\text {ref }}$ is the reflected wave, and $U_{\text {tra }}$ is the wave passing through, $R_{u}$ and $R_{d}$ are the impedances of the coaxial line upstream and downstream to the gap, respectively, and

$$
R_{\text {cell }}=Z_{B}\left\|Z_{C}\right\| Z_{F}
$$

$Z_{C}, Z_{F}$, and $Z_{B}$ are defined as in Eq. (1).

There should be no reflection from downstream when

$$
R_{u}=R_{d}+R_{\text {cell }} .
$$

On account of the escalating high voltage, matched conducting stalks were devised as in Fig. 10. In this case there should be no reflection back to the diode. The impedances of the coaxial line are listed in Tables II and III, where $R_{\text {cell }}=8.8 \Omega$.

To simplify installation, the diameter of the cathode stalk inside the first cell is different from Table II, and 

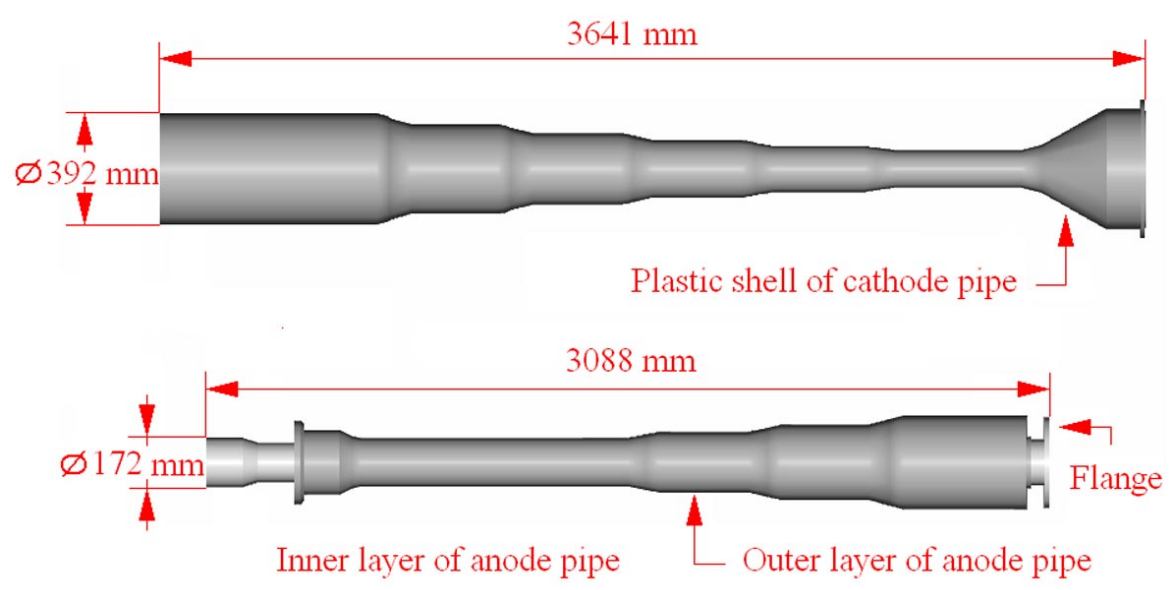

FIG. 10. (Color) Matched cathode (above) and anode (below) stalk.

near the gap a copper ring is used to connect the stalk and bore of the cell.

The anode stalk is a pipe of dual layers. The outer layer shapes with matched size and its two ends are connected with the grand potential cover board of the induction cell and anode shroud. The inner layer protrudes and is connected to the downstream cell and bore of the anode.

In fact the reflection is inevitable since $R_{F}$ changes in different excitation phase of ferrite and $R_{C}$ changes with temperature. In addition it is difficult to match every section along the transmission path of the pulse.

\section{RADIAL INSULATOR}

As mentioned before, the decline of the cathode and anode will degrade high voltage insulation and beam properties. The decline results from the following design. First, the cathode and anode stalk extend to about 3.6 and $3 \mathrm{~m}$, respectively, as shown in Fig. 10. Furthermore, the cathode and anode weigh heavily with large flat electrodes, and with magnets inside.

The radial insulator for the Dragon-I injector is designed to locate the center of cathode and anode along the mechanic axis of the whole system, and its structure and dimension are shown in Fig. 11.
Each insulator consists of 2 pieces of polymethylmethacrylate with a diameter of $1560 \mathrm{~mm}$. The thickness of the piece, with one surface opposed to oil, is about $100 \mathrm{~mm}$, and the other is about $200 \mathrm{~mm}$ whose vacuum surface is shaped like a sawtooth, with metal grading rings inserted at the end of every $45^{\circ}$ slope.

Copper sulfate solution was filled between two pieces of polymethylmethacrylate. The resistance value of the solution changes linearly with the radius according to Eq. (8) to homogenize the electric field distribution along the insulators,

$$
\Delta R=\rho \frac{\Delta r}{S},
$$

where $\Delta R$ is the change of the resistor, $\Delta r$ is the change of radius, $S$ is the area of the cross section of liquid, and $\rho$ is the resistivity. When $\Delta r \ll r, S \approx 2 \pi r \times d$, and $d$ is the width of the liquid layer. So

$$
d=\frac{\rho \Delta r}{2 \pi r \Delta R} \propto \frac{1}{r} .
$$

In addition, when connected in parallel with the diode impedance the liquid resistor functions to make the diode a matching load. The diode impedance is about $1 \mathrm{k} \Omega$, and

TABLE II. Size of the cathode stalk and corresponding impedance of the coaxial line.

\begin{tabular}{lccccccc}
\hline \hline Number of the local cell (start from the ground end) & 1 & 2 & 3 & 4 & 5 & 6 \\
\hline Impedance of the coaxial line $(\Omega)$ & 0 & 8.8 & 17.6 & 26.4 & 35.2 & 44 & 52.8 \\
Diameter of the conducting stalk $(\mathrm{mm})$ & 490 & 392 & 314 & 251 & 201 & 161 & 129 \\
\hline \hline
\end{tabular}

TABLE III. Size of the anode stalk and corresponding impedance of the coaxial line.

\begin{tabular}{lccrr}
\hline \hline Number of the local cell (start from the ground end) & 1 & 2 & 3 & 4 \\
\hline Impedance of the coaxial line $(\Omega)$ & 8.8 & 17.6 & 26.4 & 35.2 \\
Diameter of the conducting stalk $(\mathrm{mm})$ & 330 & 264 & 211 & 169 \\
\hline \hline
\end{tabular}




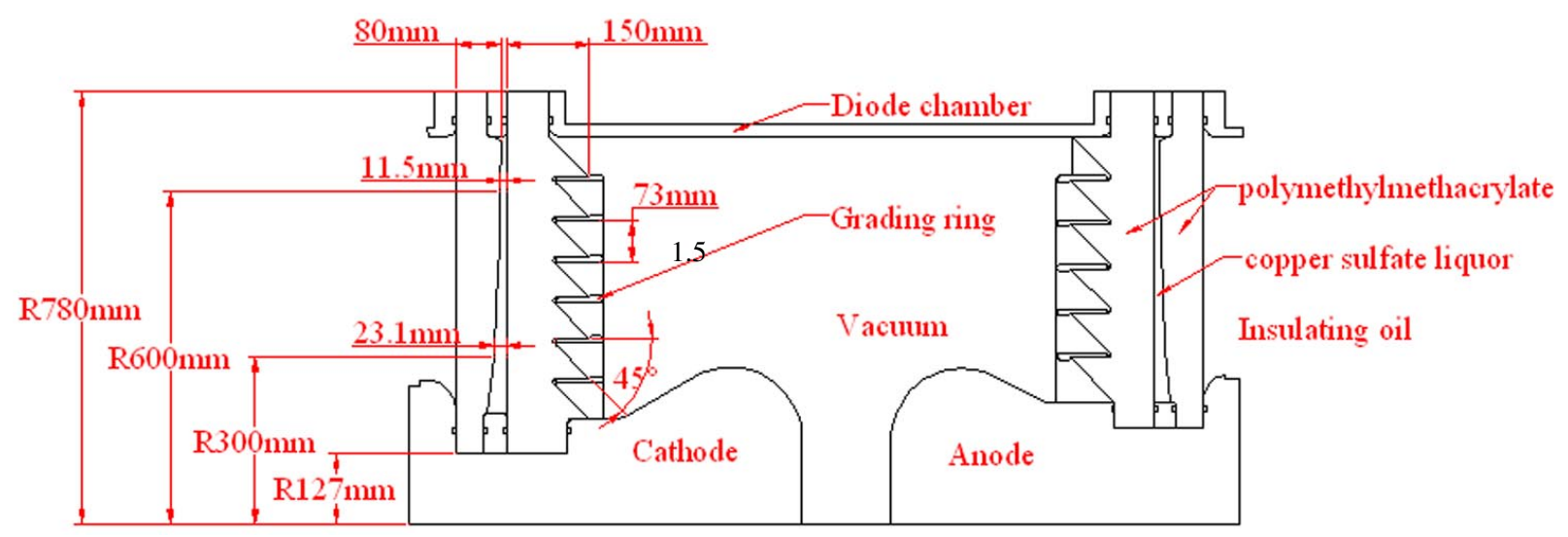

FIG. 11. (Color) Structure and dimension of the radial insulator.

the impedances of the transmission line are $52.8 \Omega$ on the side of the cathode, and $44 \Omega$ on the anode side, as shown in Tables II and III. The liquid resistor should be about 56 and $46 \Omega$ on the side of cathode and anode, respectively. In this case the current passing through the liquid resistors will be far higher than the emitting beam current, and the total power consumption would exceed the capability of the pulsed-power system, so the liquid resistor is set at about $10 \mathrm{k} \Omega$ to ensure sufficient emission.

Field simulation of the final design shows that the peak field is far lower than the surface flash limit. Under the voltage of 3.6 MV, the peak field strength of the triple point, of the cathode radial insulator, is $34 \mathrm{kV} / \mathrm{cm}$, and that of the anode insulator is $20 \mathrm{kV} / \mathrm{cm}$.

\section{TUNING RESULT}

\section{A. Beam current}

Since 2003, the injector has experienced more than 2000 shots with high reliability. When operating at $3.5 \mathrm{MV}$, with the cathode-anode gap of $170 \mathrm{~mm}$, the emitting electron beam current reaches $3.4 \mathrm{kA}$ measured with a B-dot placed in the diode zone. The output beam current is $2.8 \mathrm{kA}, 80 \mathrm{~ns}$ (FWHM) measured by the beam position monitor at the exit of the injector. The corresponding axial magnetic field distribution is shown in Fig. 12.

\section{B. Emittance}

Emittance was measured based on the pepper-pot technique first [17]. A $1.8 \mathrm{~mm}$ thick tantalum plate with an array of $1 \mathrm{~mm}$ diameter holes was used. After passing through the mask, the electrons drift for $204 \mathrm{~mm}$ and then are intercepted by a piece of thin quartz. Čerenkov radiation generated from the interaction between quartz and the electron beam was recorded by a fast frame camera. The mean emittance measured is $1495 \pi \mathrm{mm}$ mrad.

The modified three gradient method (MTGM) [18] was adopted to improve the precision of measurement since collimation and penetration of electrons through the above mask brought additional errors to the result. MTGM is

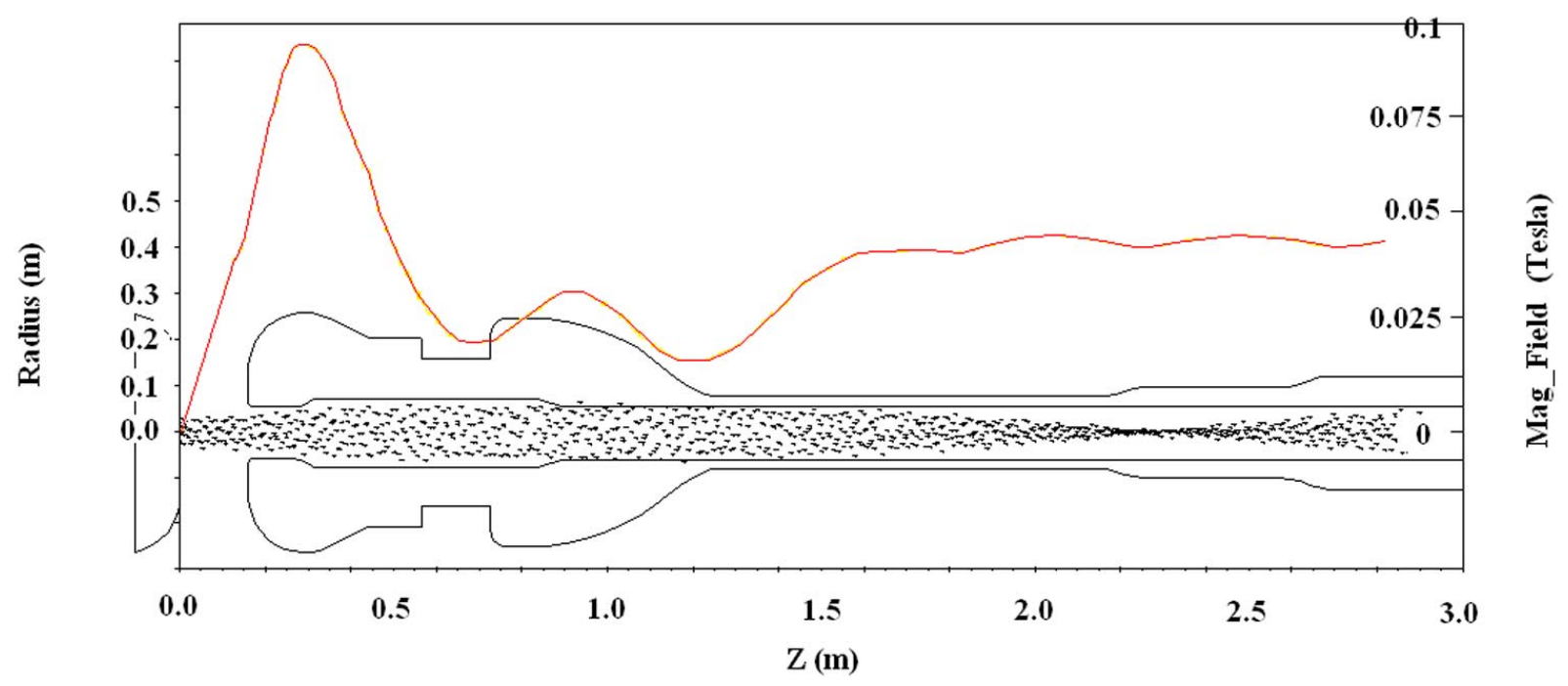

FIG. 12. (Color) Axial magnetic field distribution along the beam axis. 
TABLE IV. Properties of injectors for LIA [19-21].

\begin{tabular}{lllllll}
\hline \hline Accelerator & FXRU & ATA & LIAXF & Dragon-I & DARHT-I & AIRIX \\
\hline Beam current $(\mathrm{kA})$ & $\sim 2.3-3.4$ & 10 & 6 & 2.8 & $\sim 1.7-4$ & $\sim 1.92-3.5$ \\
Energy $(\mathrm{MeV})$ & 2.5 & 2.5 & 1 & 3.5 & $\sim 2.5-3.75$ & 3.8 \\
Normalized emittance $(\pi$ mm mrad) & 3213 & 2500 & $\ldots$ & 1040 & 1200 & $1165(3.5 \mathrm{kA})$ \\
Drive mode & IVA & IVA & IVA & IVA & PFL & PFL \\
\hline \hline
\end{tabular}

developed from traditional three gradient method beam emittance measurement. The dependence of the beam radius evolution on the focusing current can be fitted by a curve calculated from the Kapchinskij-Vladimirskij envelop equation, and the curve depends on beam radius, divergence, and beam emittance. From the relation between the beam radius and the focusing current, an approximation of the emittance can be obtained by the above fitting procedure. The measured normalized emittance of the flattop part of the beam pulse is $1040 \pi \mathrm{mm}$ mrad.

\section{Energy spread}

Energy spread was measured using a magnetic spectrometer.

The output electron beam from the injector is bent after it enters between the poles of a quarter-circular magnet. At the exit of the magnet electrons are intercepted by a piece of thin quartz, and Čerenkov light is recorded using a fast frame camera. The measurement shows that the energy spread of the flattop part of the beam pulse is $2.1 \%$ around the central energy of $3.5 \mathrm{MeV}$.

The measured energy spread is higher than expected, which may be attributed to the multipulse reflection along the pulse transmission line as mentioned in Sec. VI.

\section{CONCLUSION}

The Dragon-I injector might be of the highest energy among the injectors powered by IVA, and with preferable beam properties at the same time, as shown in Table IV.

Injectors of DARHT-I and AIRIX are powered directly by PFL, and export electron beams with higher energy up to $4 \mathrm{MeV}$. Compared to injectors driven by IVA, it is the most remarkable advantage of the injectors driven by PFL that the anode is at ground potential, which is convenient for installation, magnetic, and measurement elements placement. Furthermore, the relatively short anode pipe of $2 \mathrm{~m}$ is good for beam transportation. However those driven by PFL are of larger size, the whole injector is as long as $18 \mathrm{~m}$, with a diameter of $2 \mathrm{~m}$.

\section{ACKNOWLEDGMENTS}

We would like to thank Professor Li Jianfeng from IFP, CAEP, Professor Luo Dashi from CAEP Graduate School, and John Power and Richard S. Konecny from ANL,
U.S.A., for their assistance in polishing up this paper, and numerous colleagues for their help during the design, manufacturing, installation, and tuning of the injector.

[1] Ian D. Smith, Phys. Rev. ST Accel. Beams 7, 064801 (2004).

[2] Raymond D. Scarpetti, John K. Boyd, Gregory G. Earley, Kenneth L. Griffin, Roderick G. Kerr, Ronald Kihara, Mike M. Ong, Jan-Mark Zentler, and Norman L. Back, 11th IEEE International Pulsed Power Conference, 1997.

[3] J. T. Weir, G. J. Caporaso, F. W. Chambers, R. Kalibjian, J. Kallman, D. S. Prono, M. E. Slominski, and A. C. Paul, IEEE Trans. Nucl. Sci. NS-32, 1812 (1985).

[4] J. Deng, B. N. Ding, J. S. Shi, Y. He, J. Li, Q. Li, G. G. Cao, L. Wen, and G. S. Dai, LINAC 98, Chicago, 1998.

[5] M. J. Burns , B.E. Carlsten, T. J. T. Kwan, D.C. Moir, D. S. Prono, S. A. Watson, E. L. Burgess, H. L. Rutkowski, G. J. Caporaso, Y.-J. Chen, Y.J. (Judy) Chen, S. Sampayan, and G. Westenskow, Proceedings of the 1999 Particle Accelerator Conference, New York.

[6] M. Mouillet, R. Boivinet, F. Bombardier, J. Delvaux, E. Merle, J. C. Ribes, P. Anthouard, J. Bardy, A. Devin, and J . de Mascureau, LINAC 2000, Monterey, 2000.

[7] J. J. Deng, B. N. Ding, L. W. Zhang, H. C. Wang, G. S. Dai, N. A. Chen, Z. Y. Dai, K. Z. Zhang, J. S. Shi, W. W. Zhang, J. Li, X. P. Liu, Y. T. Xie, M. Wang, L. Wen, H. Li, J. S. Wang, Z.C. Xie, and M.H. Wang, LINAC 2002, Gyeongju, Korea.

[8] Li Hong, Liu Yunlong, Zhang Linwen, Deng Jianjun, and Yao lin, High Power Laser Part. Beams 16, 10 (2004) (in Chinese).

[9] Zhang Wenwei, Pan Haifeng, Li Hong, Liu Yunlong, and Zhang Linwen, High Power Laser Part. Beams 14, 42002 (in Chinese).

[10] G. J. Caporaso and A. G. Cole, High Current Electron Transport (American Institute of Physics, New York, 1992), p. 1623.

[11] R. J. Adler, G. F. Kiuttu, B. E. Simpkins, D. J. Sullivan, and D. E. Voss, Rev. Sci. Instrum. 56, 766 (1985).

[12] Stanley Humphries, Jr., Charged Particle Beams (John Wiley and Sons, New York, 1990), p. 263.

[13] Huacen Wang, Kai Zhi Zhang, Long Wen, Yong Zou, Qinggui Lai, Wenwei Zhang, Jianjun Deng, and Bonan Ding, Proceedings of the 1999 Particle Accelerator Conference, New York.

[14] http://www.myjiuyuan.com. 
[15] Chengjun Liu, Wenjun Zhu, Kaizhi Zhang, Wenwei Zhang, and Haijun Yu, Proceedings of the 11th International Conference on High Power Particle Beams, Prague, 1996, p. 1014.

[16] Michael Burns, Ken Chellis, Cathy Mockler, Tom Tucker, George Velasquez, and Roger Van Maren, Proceedings of the 1999 Particle Accelerator Conference, San Francisco, CA.

[17] J. de Mascureau, C. Bonnafond, A. Devin, E. Merle, G. Polyart, and D. Villate, Proceedings of 1993 IEEE Particle Accelerator Conference, pp. 2115-2117.
[18] S. Marghitu and C. Oproiu, Proceedings of the International Conference on Accelerator and Experimental Physics Control System, 2001.

[19] A.C. Paul, UCRL-TR-203991, 2004.

[20] Carol Wilkinson, http://www.ligo.caltech.edu/docs/G/ G030500-00/G030500-00.pdf.

[21] E. Merle, R. Boivinet, M. Mouillet, J. C. Picon, O. Pierret, Ph. Anthouard, J. Bardy, C. Bonnafond, A. Devin, and P. Eyl, Proceedings of the 1999 Particle Accelerator Conference, New York. 